\title{
Myeloid SOCS3 Deficiency Regulates Angiogenesis via Enhanced Apoptotic Endothelial Cell Engulfment
}

\author{
Irina Korovina ${ }^{\mathrm{a}, \mathrm{b}} \quad$ Ales Neuwirth $^{\mathrm{a}}$ David Sprott ${ }^{\mathrm{a}} \quad$ Maria Troullinaki $^{\mathrm{a}}$ \\ David M. Poitz ${ }^{\mathrm{a}}$ Andreas Deussen ${ }^{c}$ Anne Klotzsche-von Ameln ${ }^{\mathrm{a}, \mathrm{c}}$ \\ a Institute for Clinical Chemistry and Laboratory Medicine, Faculty of Medicine, Technische Universität Dresden, \\ Dresden, Germany; ${ }^{\mathrm{b}}$ OncoRay, National Center for Radiation Research in Oncology, Faculty of Medicine, Technische \\ Universität Dresden, Dresden, Germany; ${ }^{C}$ Institute of Physiology, Faculty of Medicine, Technische Universität \\ Dresden, Dresden, Germany
}

\section{Keywords \\ Suppressor of cytokine signaling $3 \cdot$ Phagocytosis . \\ Angiogenesis · Growth arrest-specific $6 \cdot$ Mer}

\begin{abstract}
Mononuclear phagocytes, such as macrophages and microglia, are key regulators of organ homeostasis including vascularization processes. Here, we investigated the role of the suppressor of cytokine signaling 3 (SOCS3) in myeloid cells as a regulator of mononuclear phagocyte function and their interaction with endothelial cells in the context of sprouting angiogenesis. As compared to SOCS3-sufficient counterparts, SOCS3-deficient microglia and macrophages displayed an increased phagocytic activity toward primary apoptotic endothelial cells, which was associated with an enhanced expression of the opsonin growth arrest-specific 6 (Gas6), a major prophagocytic molecule. Furthermore, we found that myeloid SOCS3 deficiency significantly reduced angiogenesis in an ex vivo mouse aortic ring assay, which could be reversed by the inhibition of the Gas6 receptor Mer. Together, SOCS3 in myeloid cells regulates the Gas6/Merdependent phagocytosis of endothelial cells, and thereby angiogenesis-related processes. Our findings provide novel
\end{abstract}

insights into the complex crosstalk between mononuclear phagocytes and endothelial cells, and may therefore provide a new platform for the development of new antiangiogenic therapies.

(C) 2019 The Author(s) Published by S. Karger AG, Basel

\section{Introduction}

Mononuclear phagocytes, including microglia and macrophages, contribute to the maintenance of tissue integrity via the phagocytic elimination of dead cells and cellular debris, a process called efferocytosis. During efferocytosis, phagocytic cells recognize membrane-associated or soluble "eat-me" signals on dying cells. Thereafter, the phagocyte engulfs the apoptotic cell, and thereby prevents the exposure of the surrounding tissue to harmful intracellular components [1-3].

Mononuclear phagocytes may regulate angiogenic processes via different mechanisms [4-6]. For instance, microglia and macrophages establish direct cell-cell interactions with endothelial cells facilitating vascular anastomosis [7], and they can remodel the extracellular ma-

Dr. Anne Klotzsche-von Ameln 
trix, for example, by secretion of membrane bound and/ or soluble proteases, which may also mobilize proangiogenic growth factors embedded in the perivascular matrix $[5,8]$. Additionally, mononuclear cells can also produce and secrete diverse pro- and antiangiogenic factors (e.g., VEGF, PDGF, TNFa, IL10, IL1 $\beta$, etc.) affecting migration, proliferation, and survival of endothelial cells $[5,8$, 9]. Multiple pathological situations accompanied by altered angiogenesis may display the rapid accumulation of cellular debris as well as activation of microglia/macrophages $[6,10]$. Recently, we could even show that mononuclear phagocytes can contribute to pathological retina angiogenesis by the modulation of endothelial cell apoptosis [11]. Subsequently, these mononuclear phagocytes may also contribute to the clearance of apoptotic endothelial cells. For instance, Poché et al. [12] observed that retinal macrophages are able to engulf endothelial cell membrane particles during the process of pupillary membrane capillary regression. However, a potential link between efferocytosis and sprouting angiogenesis has not been addressed yet.

To this end, we engaged here myeloid deficiency of suppressor of cytokine signaling 3 (SOCS3), a negative regulator of cytokine signaling pathways (IL6, IL10, IFNgamma) and modulator of macrophage activation [1316]. As demonstrated recently, SOCS3 silencing in human monocyte-derived macrophages increased the phagocytic capacity toward apoptotic neutrophils [17]. In the current study, we tested whether SOCS3 in mononuclear phagocytes (microglia and macrophages) might regulate their phagocytic activity toward apoptotic endothelial cells and whether this function could potentially control angiogenic sprouting.

\section{Materials and Methods}

\section{Animals}

Mice expressing Cre-recombinase under the control of the LysM promoter [18] were crossed with $\mathrm{SOCS}^{\mathrm{fL} / \mathrm{fL}}$ mice [19] to generate mice with SOCS3 deletion in cells of the myeloid lineage (LysM-Cre SOCS3 ${ }^{\mathrm{fL} / \mathrm{fL}}$ ). Mice were housed at the Experimental Center of the Medical Faculty of the Technische Universität Dresden.

\section{Aortic Ring Assay}

The aortic ring assay was performed according to a previously described protocol with some modifications [20]. Thoracic aortas from euthanized LysM-Cre SOCS3 $3^{\mathrm{fL} / \mathrm{fL}}$ and $\mathrm{SOCS} 3^{\mathrm{fL} / \mathrm{fL}}$ mice were dissected, cleaned of surrounding adipose tissue and cut into rings of $0.5 \mathrm{~mm}$ width. After $24 \mathrm{~h}$ incubation in serum-free Opti-MEM medium (Thermo Fisher Scientific) with antibiotics $(100 \mu \mathrm{g} / \mathrm{mL}$ streptomycin, $100 \mathrm{U} / \mathrm{mL}$ penicillin), aortic rings were embedded in a collagen matrix $(1 \mathrm{mg} / \mathrm{mL}$ type I collagen, Thermo Fisher Scientific) diluted in DMEM medium and grown at $37^{\circ} \mathrm{C}$ in a humidified 5\% $\mathrm{CO}_{2}$ incubator for 5-7 days. In some experiments, the embedded rings were treated for 5 days with $2 \mu \mathrm{g} / \mathrm{mL}$ mouse MerFc (R\&D Systems, Wiesbaden, Germany) or control IgG1-Fc (R\&D Systems, Wiesbaden, Germany) in Opti-MEM medium, supplemented with $2.5 \%$ FBS. For macrophage depletion, aortic rings from wild-type mice were incubated overnight with either $10 \%$ PBS-liposomes or $10 \%$ clodronate-liposomes (clodronateliposomes.com). The next day rings were washed twice for $1 \mathrm{~h}$ in Opti-MEM medium, embedded in collagen and cultured for 5 days.

After incubation, aortic rings were fixed with $10 \%$ formalin solution (Sigma-Aldrich, Steinheim, Germany) for $30 \mathrm{~min}$ at room temperature (RT). Rings were washed three times with PBS and permeabilized in $0.25 \%$ Triton X-100 solution diluted in PBS for 1 $\mathrm{h}$ at RT. Following permeabilization, FITC-conjugated Bandeirea Simplicifolia isolectin B4 $(10 \mu \mathrm{g} / \mathrm{mL}$, Sigma-Aldrich, Steinheim, Germany) was applied and incubated overnight at $4^{\circ} \mathrm{C}$. For CD 45 staining, an additional overnight incubation with an antimouse PE-conjugated CD45 antibody (1:100, Clone 30-F11, BD Pharmingen) was performed.

At the final step, aortic rings were washed with PBS and scanned with an Olympus IX 83 spinning disk microscope (Olympus, Hamburg, Germany) equipped with a Yokogawa CSU-X1 Spinning Disk Unit and Andor iQ 3.2 software. Image analysis was performed using Fiji [21] and AngioTool Software (version 0.6a) [22]. Immunofluorescence images (gray scale values) were inverted to enhance the visibility of sprouts [23].

\section{Human Retinal Microvascular Endothelial Cells}

Human retinal microvascular endothelial cells (HRMECs), purchased from Cell Systems (Kirkland, WA, USA), were seeded on gelatin pretreated plates and grown in Endothelial Cell Growth Medium (PromoCell, Heidelberg, Germany) supplemented with $10 \%$ FBS and antibiotic cocktail $(100 \mu \mathrm{g} / \mathrm{mL}$ streptomycin, $100 \mathrm{U} /$ $\mathrm{mL}$ penicillin) at $37^{\circ} \mathrm{C}$ in a humidified $5 \% \mathrm{CO}_{2}$ incubator.

\section{Primary Microglia Culture}

Microglia cells were isolated from the mouse brain according to a previously described protocol with modifications [24]. Briefly, after euthanasia, brains were removed and dissected on ice. Homogenized tissue was incubated in enzymatic solution containing $0.1 \mathrm{mg} / \mathrm{mL}$ papain (Sigma-Aldrich, Steinheim, Germany), $1 \mathrm{mg} /$ $\mathrm{mL}$ collagenase type I (Thermo Fisher Scientific), $1.2 \mathrm{U} / \mathrm{mL}$ dispase II (Sigma-Aldrich, Steinheim, Germany), $1 \mathrm{mM} \mathrm{L-cystein}$ (Sigma-Aldrich, Steinheim, Germany), and $0.1 \mathrm{mg} / \mathrm{mL}$ DNAse I (Roche, Mannheim, Germany) in DMEM high glucose medium (Thermo Fisher Scientific) for $40 \mathrm{~min}$ at $37^{\circ} \mathrm{C}$ under continuous stirring. The dissociated tissue was filtered through the cell strainer (pore size $100 \mu \mathrm{m}$ ) and centrifuged for $7 \mathrm{~min}$ at a speed of $300 \mathrm{~g}$. The supernatant was carefully removed, and the pellet was resuspended in $10 \mathrm{~mL}$ of growth medium DMEM F/12 with GlutaMAX ${ }^{\mathrm{TM}}$ supplement (Thermo fisher scientific) to wash out the residues of the enzymatic solution. After the second centrifugation round (300 $\mathrm{g}, 5 \mathrm{~min}$ ), dissociated tissue was seeded on a poly-Llysine (Sigma-Aldrich, Steinheim, Germany) precoated plates and cultured in DMEM/F12 with $10 \%$ FBS, $100 \mu \mathrm{g} / \mathrm{mL}$ streptomycin, $100 \mathrm{U} / \mathrm{mL}$ penicillin, and freshly added $5 \mathrm{ng} / \mathrm{mL}$ GM-CSF (PeproTech, Hamburg, Germany) at $37^{\circ} \mathrm{C}$ in a humidified $5 \% \mathrm{CO}_{2}$ incu- 
bator. Growth medium was replaced every 2-3 days, and detached microglial cells were harvested and plated onto poly-L-lysine precoated plates.

\section{Isolation of Bone Marrow-Derived Macrophages}

Bone marrow-derived macrophages (BMDMs) were isolated from the mice according to a previously described protocol [25]. The femurs and tibias were removed and cleaned of surrounding muscles. At the next step, bone marrow was flushed out of the bones using a $30 \mathrm{G}$ needle with sterile PBS. Then bone marrow suspension was filtered through a $40 \mu \mathrm{m}$ strainer into a fresh tube and centrifuged for $5 \mathrm{~min}$ at $130 \mathrm{~g}$ at $4^{\circ} \mathrm{C}$. Following centrifugation, the pellet was incubated in RBC lysis buffer (eBioscience) for $1 \mathrm{~min}$. Bone marrow cells were then resuspended in RPMI 1640 medium (Thermo Fisher Scientific) with $10 \%$ FBS, $100 \mu \mathrm{g} / \mathrm{mL}$ streptomycin, $100 \mathrm{U} / \mathrm{mL}$ penicillin, and freshly added $5 \mathrm{ng} / \mathrm{mL}$ GM-CSF. BMDMs were seeded on a Petri dish and incubated for 6 days at $37^{\circ} \mathrm{C}$ in a humidified $5 \% \mathrm{CO}_{2}$ incubator.

\section{Induction of Apoptosis in HRMEC by Staurosporine}

Apoptosis induction in endothelial cells was performed by using the natural metabolite staurosporine (Sigma-Aldrich, Steinheim, Germany) [26]. Upon reaching 80\% confluency, HRMECs were washed 3 times with sterile PBS and treated with staurosporine at a concentration of $200 \mathrm{nM}$ for $12 \mathrm{~h}$ in serum-free RPMI-1640 medium (Thermo Fisher Scientific). After this incubation period, all cells (including the floating) were used for the phagocytosis assay.

\section{Phagocytosis Assay}

To analyze the phagocytic activity of myeloid cells toward endothelial cells we cocultured brain-derived mouse microglia or BMDM and apoptotic HRMEC labeled with $2^{\prime}, 7^{\prime}$-bis-(2carboxyethyl)-5-(and-6)-carboxyfluorescein acetoxymethyl ester (BCECF-AM, Thermo Fisher Scientific). To perform BCECF-labeling of HRMEC, approximately $5 \times 10^{5}$ live cells were washed with PBS and centrifuged at $300 \mathrm{~g}$ for $5 \mathrm{~min}$. The supernatant was discarded, and the cell pellet was resuspended in sterile PBS with BCECF-AM at a final concentration of $4 \mu \mathrm{mol} / \mathrm{L}$ for $15 \mathrm{~min}$ at $37^{\circ} \mathrm{C}$. Then apoptosis induction was performed as described above. Afterward, HRMECs were stained again with BCECF-AM and used for the experiment.

In phagocytosis-blocking experiments, phagocytes were preincubated with recombinant mouse Mer-Fc or IgG1 Fc as a control at concentration $400 \mathrm{ng} / \mathrm{mL}$ for $1 \mathrm{~h}$ prior to the phagocytosis assay. Apoptotic HRMECs were mixed with BMDM or microglia in the ratio $1: 1$ and cocultured for $4 \mathrm{~h}$ at $37^{\circ} \mathrm{C}$. Then the cells were fixed with $10 \%$ formalin solution for $10 \mathrm{~min}$ at $\mathrm{RT}$, washed, and stained with anti-mouse PE-conjugated CD45 antibodies (1:400, Clone 30-F11, BD Pharmingen). Imaging was performed using an Axiovert 200 Inverted Fluorescence Microscope and Zen 2 (blue edition) image processing software (Carl Zeiss). Phagocytic index of $\mathrm{BMDM} /$ microglia was expressed as a percentage of phagocytes containing endothelial cells and/or endothelial cell particles. At least 500 mononuclear phagocytes per isolation were analyzed. Cell size of microglia was defined with Fiji in CD45-stained microglia [21].

RNA Extraction, cDNA Synthesis, and Quantitative PCR

RNA extraction from cultured cells and reverse transcription were performed using NucleoSpin RNA mini-kit (Macherey Na- gel, Düren, Germany) and iScript cDNA Synthesis Kit (Bio-Rad, Munich, Germany), respectively, according to the manufacturer's protocol. The expression levels of genes of interest were estimated using quantitative PCR. For each reaction, 20-30 ng of template cDNA were mixed with gene-specific primers (Life Technologies) and SsoFast EvaGreen supermix (Bio-Rad, Munich, Germany) using a RealTime-PCR Cycler (Bio-Rad, Munich, Germany). Primer pairs, listed below, were designed using the web program Primer 3 Input (version 0.4.0). Primer sequences: 18S-Fw: GTTCCGACCATAAACGATGCC, 18S-Rev: TGGTGGTGCCCTTCCGTCAAT,TBP[TATA-boxbindingprotein]-Fw:TCTACCGTGAATCTTGGCTGTAAA, TBP-Rev: TTCTCATGATGACTGCAGCAAA, growth arrest-specific 6 [Gas6]-Fw: TGCTGGCTTCCGAGTCTTC, Gas6-Rev: CGGGGTCGTTCTCGAACAC, milk fat globule-EGF factor 8 [Mfge8]-Fw: GGGCATCCACTGTGAAACCGGTT, Mfge8-Rev: CATCTTCCGCAGAAGGTTCACCTGG, Il1 $\beta$-Fw: GCTGAAAGCTCTCCACCTCA, Il1 $\beta$-Rev: AGGCCACAGGTATTTTGTCG, Il6-Fw: CAAAGCCAGAGTCCTTCAGAG, Il6-Rev: TGGTCCTTAGCCACTCCTTC, Il10-Fw: ATCGATTTCTCCCCTGTGAA, Il10-Rev: TGTCAAATTCATTCATGGCCT, Tnfa-Fw: CGAGTGACAAGCCTGTAGCC, Tnfa-Rev: GGTTGTCTTTGAGATCCATGC, Mcp1-Fw: AGGTCCCTGTCATGCTTCTG, Mcp1-Rev: GCTGCTGGTGATCCTCTTG, Rantes-Fw: CCCTCACCATCATCCTCACT, Rantes-Rev: GAGCACTTGCTGCTGGTGTA, Vegf-Fw: AGTCCCATGAAGTGATCAAGTTCA and Vegf-Rev: ATCCGCATGATCTGCATGG. Analysis of relative gene expression was performed using $2^{-\Delta \Delta C t}$ method described by Livak and Schmittgen [27].

\section{ELISA}

To estimate Gas6 production by myeloid cells, we utilized the commercially available Mouse Gas6 ELISA kit (Abcam, Cambridge, UK). Primary microglial cells were incubated for $6 \mathrm{~h}$ in growth medium DMEM/F-12 containing $0.5 \%$ FBS at $37^{\circ} \mathrm{C}$ in a humidified $5 \% \mathrm{CO}_{2}$ incubator. Following an incubation step, supernatants were collected and analyzed by ELISA according to the manufacturer's protocol. Final results were normalized to the total protein concentration in each well, defined by BCA protein assay kit (Thermo Fisher Scientific).

\section{Statistical Analysis}

The statistical analysis was performed using GraphPad Prism 6 software. Data are presented as the mean \pm SEM. The statistical significance of data was determined using two-tailed unpaired Student $t$ test.

\section{Results}

\section{SOCS3-Deficient Mononuclear Phagocytes}

Demonstrate an Increased Phagocytic Activity Toward Apoptotic Endothelial Cells

First, we investigated the functional properties of SOCS3-deficient mononuclear phagocytes isolated from mice with myeloid-specific SOCS3 deficiency, which were generated by crossing SOCS3 floxed mice with Cre-mice carrying Cre recombinase under the con- 


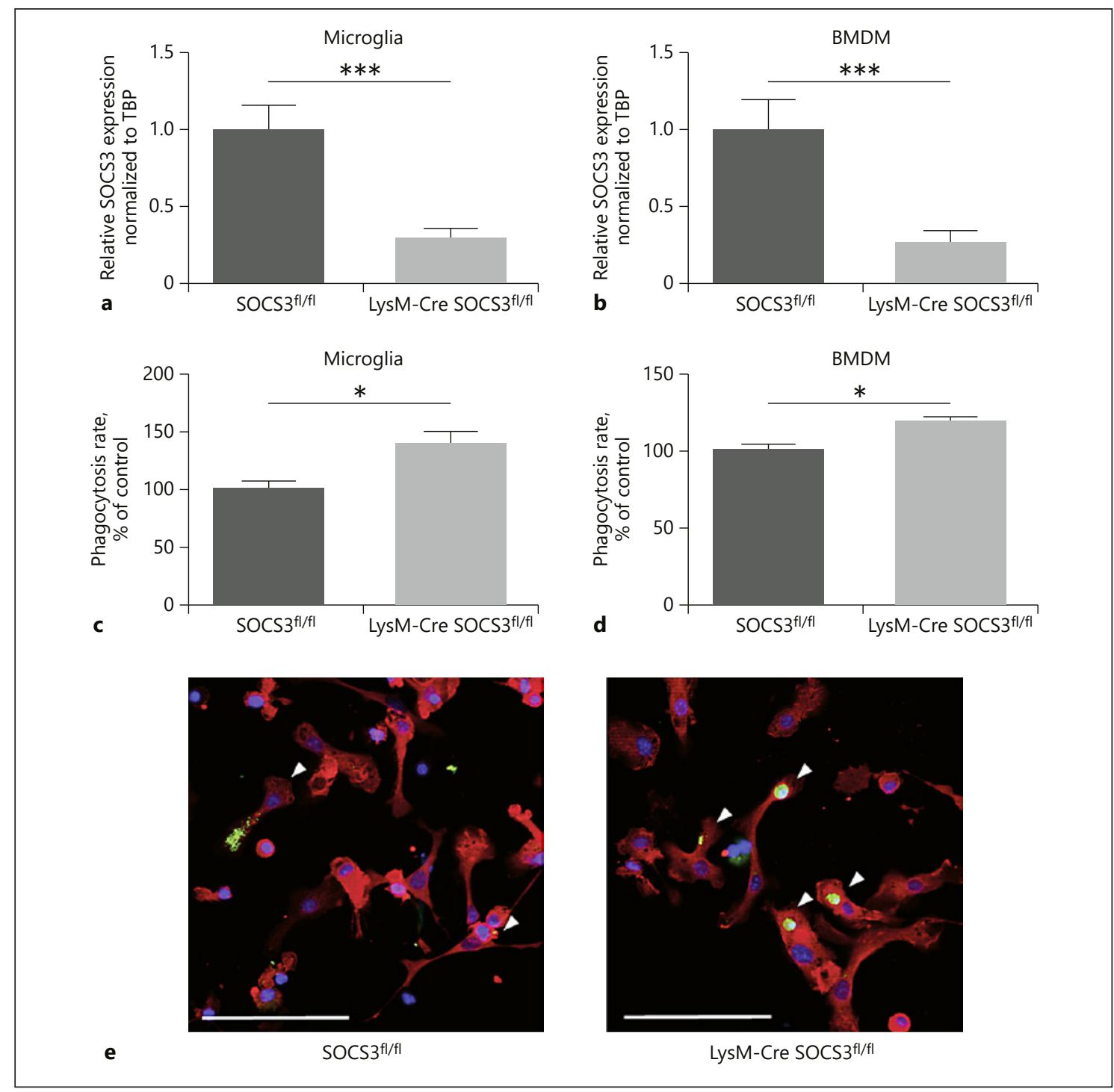

Fig. 1. SOCS3-deficient mononuclear phagocytes demonstrate enhanced phagocytic properties toward apoptotic endothelial cells. a, b Quantitative PCR analysis for SOCS3 mRNA expression of brain-derived microglia $(n=11 ; \mathbf{a})$ and $\operatorname{BMDM}(n=11 ; \mathbf{b})$. Phagocytic activity toward apoptotic retinal endothelial cells was assessed in myeloid SOCS3-deficient (LysM-Cre SOCS3 ${ }^{\mathrm{fL} / \mathrm{fL}}$ ) and wild-type SOCS3-sufficient (SOCS3 $3^{\mathrm{fL} / \mathrm{fL}}$ ) primary microglia (c) and BMDM (d). Phagocytosis rate is expressed as \% of control. Phagocytosis rate of wild-type primary microglia and BMDM was set as the $100 \%$ control $(n=11$ independent microglia isolations

trol of the LysM-cre promoter $[18,19]$. We isolated 2 types of mononuclear phagocytes, namely, primary brain microglia and BMDM. In all experiments, we compared cells derived from Cre-negative (SOCS3 ${ }^{\mathrm{fL} / \mathrm{fL}}$ ) with Cre-positive (LysM-CreSOCS3 ${ }^{\mathrm{fL} / \mathrm{fL}}$ ) cells. As shown in Figure 1a and b, SOCS3 expression was reduced to per group and $n=4$ independent BMDM isolations per group). e Representative images depicting coculture of primary microglia stained with a CD45-PE antibody (red) and BCECF-labeled apoptotic endothelial cells (green). Colocalization of both fluorochromes appears yellow. White arrowheads indicate phagocytes with incorporated apoptotic endothelial cells or endothelial cell particles. Nuclei were stained with DAPI. Data are shown as mean \pm SEM. ${ }^{*} p<0.05$ and ${ }^{* * *} p<0.001$. The scale bar on e: $100 \mu \mathrm{m}$. SOCS3, suppressor of cytokine signaling 3; BMDM, bone marrowderived macrophage.
$70 \%$ in microglia and $73 \%$ in BMDM as compared with SOCS3-proficient control cells. Of note, several important cytokines and growth factors were not altered owing to SOCS3 deficiency (online suppl. Fig. 1a; see www. karger.com/doi/10.1159/000502645 for all online suppl. material). To investigate the efferocytic activity of these 


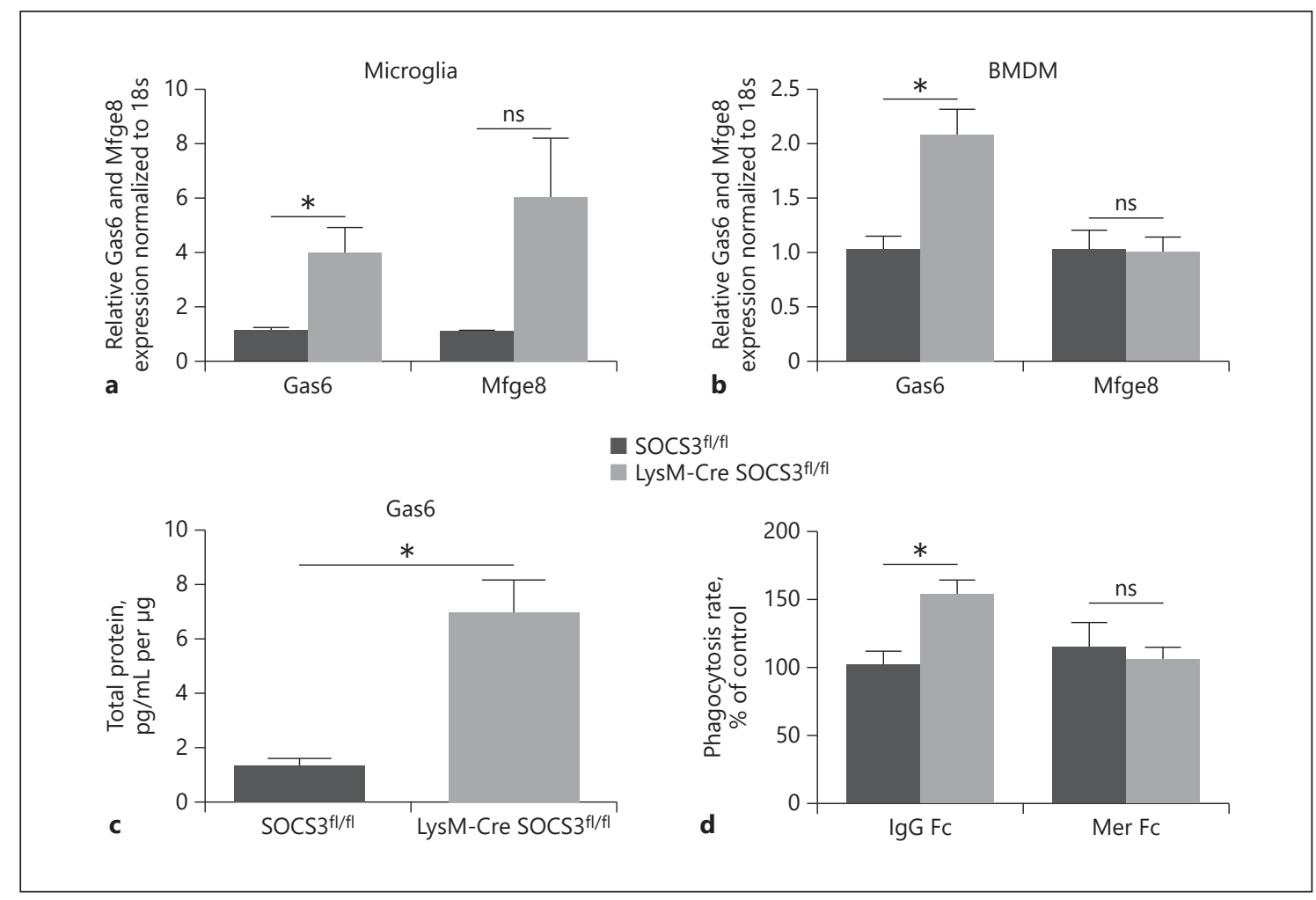

Fig. 2. Enhanced production of Gas6 by SOCS3-deficient microglia contributes to increased phagocytic activity. Relative mRNA expression of Gas6 and MFG-E8 in primary microglia (a) and $\operatorname{BMDM}(\mathbf{b} ; n=4$ independent microglia isolations per group, $n=$ 5-10 independent BMDM isolations per group). Relative mRNA expression of Gas6 and MFG-E8 in wild-type cells was set as 1 . c ELISA revealing the upregulation in the Gas6 level in cell culture supernatants from primary microglia $(n=3-5$ independent cell isolations per group). d SOCS3-deficient and SOCS3-sufficient

cells toward endothelial cells, we established a coculture model, including BCECF-labeled apoptotic retinal microvascular endothelial cells (HRMEC) and phagocytes (primary brain microglia or BMDM) and quantified endothelial phagocytosis. Interestingly, the amount of engulfed endothelial cells/endothelial cell particles was significantly increased in SOCS3-deficient microglia and BMDM, suggesting that SOCS3-deficiency enhanced their efferocytic function (Fig. 1c, d). The increased uptake of labeled endothelial cells/endothelial cell particles is also evident from the micrograph (Fig. 1e). In line with the data from Gordon et al. [17], we observed that SOCS3-deficient microglia cells showed a significant increase in cell size as compared to the control cells (online suppl. Fig. 1b). primary microglia were treated with recombinant Mer-Fc or IgG Fc as control and phagocytosis of apoptotic endothelial cells was assessed. The phagocytosis rate is expressed as \% of control; phagocytosis rate of wild-type cells in the presence of $\operatorname{IgG~Fc}$ was set as $100 \%$ ( $n=4-6$ independent cell isolations per group). All data are shown as mean \pm SEM, ${ }^{*} p<0.05$. ns, non-significant; Mfge8, milk fat globule-EGF factor 8; Gas6, growth arrest-specific 6; SOCS3, suppressor of cytokine signaling 3; BMDM, bone marrow-derived macrophage.

The Increased Phagocytic Activity of SOCS3-Deficient Mononuclear Phagocytes Is Mediated by the Gas6/

Mer Pathway

To elucidate the mechanism of phagocytosis activation in SOCS3-deficient mononuclear phagocytes, we analyzed the expression of factors involved in efferocytosis.

We found that SOCS3-deficient mononuclear phagocytes (both primary microglia and BMDM) displayed a significantly increased expression of Gas6, a secreted bridging molecule (opsonin), which promotes the engulfment of apoptotic cells $[28,29]$, at the mRNA and protein level (Fig. 2a-c). In contrast, we found a tendency for the upregulation of the opsonin Mfge8 only in microglia cells but not in BMDM (Fig. 2a, b).

We next assessed the functional contribution of Gas6 to the enhanced phagocytosis of apoptotic endothelial 
cells by SOCS3-deficient mononuclear phagocytes, as compared to SOCS3-sufficient cells. To this end, we inhibited the activity of the Gas6 receptor Mer in primary mouse microglia with a chimeric recombinant protein Mer-Fc. An inhibitory effect of the soluble Mer toward the membrane-bound Mer has been shown in previous studies [30, 31]. Interestingly, the elevated phagocytosis of apoptotic endothelial cells by SOCS3-deficient microglia was reversed by Mer-Fc (Fig. 2d), as compared to the control Fc treated group. Thus, increased Gas6 production in SOCS3-deficient mononuclear phagocytes accounts for the enhanced apoptotic endothelial cell engulfment by SOCS3-deficient mononuclear phagocytes.

\section{SOCS3 Deficiency in Mononuclear Phagocytes}

Decreases Sprouting Angiogenesis in the ex vivo Aortic Ring Assay in a Manner Dependent on the Gas6/Mer Interaction

Since SOCS3-deficient microglia/macrophages demonstrated enhanced elimination of apoptotic endothelial cells, we addressed whether this effect might influence angiogenesis-related processes. To this end, we utilized an aortic ring assay as an ex vivo angiogenesis model [20]. We isolated aortic rings from myeloid-specific SOCS3-deficient and SOCS3-sufficient mice. Aortic explants contain endothelial and nonendothelial cells including adventitial macrophages, which migrate into the periaortic space prior to the formation of vascular outgrowths. Macrophages are located in close contact to the angiogenic sprouts (online suppl. Fig. 2a) and were shown to be involved in the regulation of neovessel formation [32,33]. According to previous reports $[32,34]$ pharmacologic ablation of phagocytes by clodronate liposomes from aortic explants blocked the formation of neovascular sprouts in the aortic ring assay (online suppl. Fig. $2 \mathrm{~b}$ ) pointing to the importance of these cells to angiogenic sprouting.

We found that the number of microvessels in aortic ring explants derived from myeloid SOCS3-deficient mice was significantly decreased, as compared to explants from SOCS3-sufficient mice (Fig. 3a, b). To clarify whether the angiogenesis-regulatory effect of SOCS3 deficiency in myeloid cells was mediated by the upregulation of Gas6-dependent efferocytosis, we blocked the interaction of Gas6 with its receptor, the tyrosine kinase Mer [29] in the aortic ring assay. To this end, aortic rings isolated from myeloid-specific SOCS3-deficient and -sufficient mice were therefore treated with a chimeric recombinant protein Mer-Fc or IgG1 Fc as a control. Interestingly, the addition of Mer-Fc reversed the decreased vascular sprouting due to myeloid-specific SOCS3 deficiency
(Fig. 3c, d). Hence, our data demonstrate that the effect of the myeloid SOCS3 on angiogenic sprouting results from the regulation of Gas6 expression, which appears to be linked to an altered phagocytic activity.

\section{Discussion}

Efficient removal of damaged and/or dead cells by "professional phagocytes" such as microglia and macrophages is a critical process for maintaining tissue homeostasis. Several previous studies suggest that a close interplay between myeloid and vascular components exists in different settings that may involve inflammation, cell death, and clearance of dead cells $[5,6,11,35]$. Here, we demonstrate a role of SOCS3 in mononuclear phagocytes for the regulation of efferocytosis of apoptotic/dead endothelial cells, which can modulate ex vivo angiogenesis. Specifically, SOCS3-deficient mononuclear phagocytes (microglia and BMDM) demonstrated enhanced phagocytic activities toward apoptotic endothelial cells, as compared to wild-type phagocytes. These data are consistent with the study published by Gordon et al. [17] showing enhanced efferocytosis of apoptotic neutrophils or phosphatidyl-serine (PS)coated beads upon SOCS3-knockdown in human macrophages. In addition, as shown before [17], we observed that SOCS3-deficient microglia cells showed a significant increase in cell size as compared to control cells. This correlates with the observation that activated and phagocytic microglia display an enlarged cell body [36].

We could further show that SOCS3-deficient mononuclear phagocytes displayed elevated expression of the opsonin Gas6, which serves as a bridging molecule between the phagocyte receptor Mer and PS on apoptotic cells, thereby promoting engulfment of the latter [28]. Alterations in mRNA expression of Gas6 indicate a potential transcriptional regulation of Gas6. Previous reports identified SOCS3 protein as a suppressor of cytokine signaling pathways via the direct inhibition of the transcription factor STAT3 [37].

Interestingly in a transcription factor dataset we could identify Gas6 as one of the target genes of the STAT3 transcription factor [38]. Given these data, we hypothesize that SOCS3 deficiency in myeloid cells might contribute to the activation of STAT3, which in turn promotes Gas6 mRNA expression.

We next demonstrated that treatment with soluble Mer-Fc (interfering with the Gas6/Mer interaction) reversed the prophagocytic effect associated with myeloidspecific SOCS3 deficiency (Fig. 2d). These findings are in 


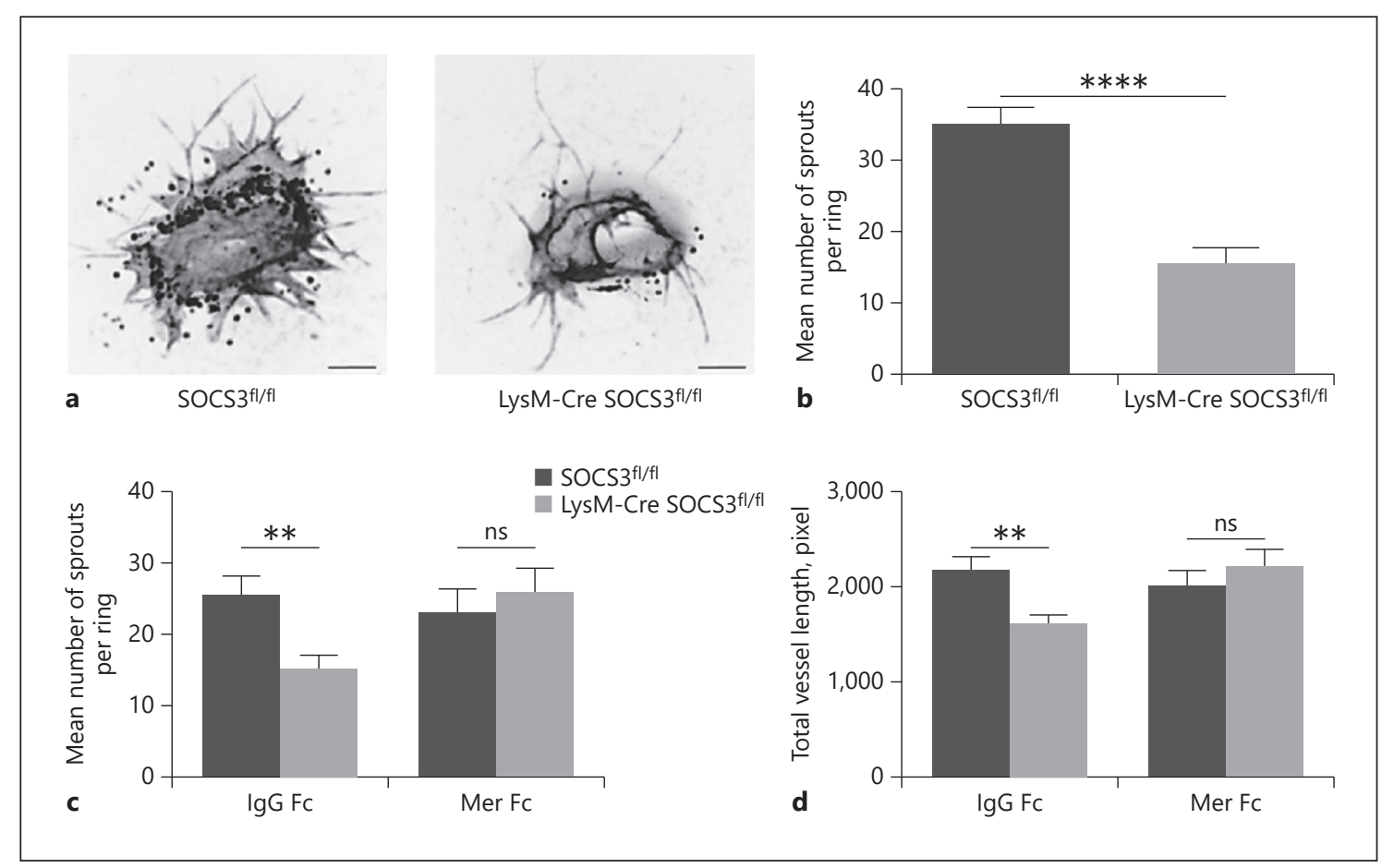

Fig. 3. SOCS3 deficiency in mononuclear phagocytes decreases endothelial cell sprouting in the mouse ex vivo aortic ring model through the Gas6/Mer axis. Mouse aortic rings derived from myeloid SOCS3-deficient (LysM-Cre SOCS3 ${ }^{\mathrm{fL} / \mathrm{fL}}$ ) and SOCS3-sufficient $\left(\mathrm{SOCS} 3^{\mathrm{fL} / \mathrm{fL}}\right.$ ) mice were embedded into collagen matrix. Isolectin B4 staining was performed to visualize microvessel formation. a Representative inverted immunofluorescence images (grayscale values) of mouse aortic rings. Scale bar $250 \mu \mathrm{m}$. b Quantitative analysis of the vascular outgrowth shown as mean number of sprouts per ring ( $n=34-40$ aortic rings per group). Data are mean \pm SEM, ${ }^{* * * *} p<0.0001$. c, d Aortic rings from myeloid SOCS3-deficient and SOCS3-sufficient mice were cultured in the presence of Mer-Fc or IgG Fc. Shown is the quantification of vascular sprouts treated with IgG Fc or Mer-Fc represented as the mean number of sprouts per ring (c) and the mean length of sprouts per aortic ring $(\mathbf{d} ; n=21-23$ aortic rings per group). Data are mean $\pm \mathrm{SEM},{ }^{* *} p<0.01$. ns, non-significant; SOCS3, suppressor of cytokine signaling 3. line with previous work showing that the phagocytic activity of macrophages for apoptotic Jurkat cells was markedly increased in the presence of recombinant Gas6, whereas the additional treatment with Mer-Fc ablated this Gas6-mediated efferocytosis [39].

Previous reports provide evidence for an essential role of phagocytosis in the regulation of physiological angiogenic processes, for example, in the regression of transient vessel networks, such as the hyaloid vessels and the pupillary membrane capillaries [40], or in the elimination of wound blood vessels [6]. In addition, it has been observed that various human diseases, such as diabetic retinopathy and cancer, are associated with accumulation of apoptotic endothelial cells [35], suggesting an involvement of mononuclear phagocytes in the regulation of angiogenic processes.

In our study, we engaged the ex vivo mouse aortic ring assay and found a significant reduction in the number of vascular sprouts due to myeloid SOCS3 deficiency. Interestingly, the treatment of the aortic explants with recombinant Mer-Fc reversed the antiangiogenic phenotype caused by SOCS3 deficiency, thereby suggesting an involvement of SOCS3 in the Gas6/Mer-dependent efferocytic pathway in sprouting angiogenesis. Remarkably, the levels of important cytokines and growth factors implicated in the regulation of angiogenesis (e.g., IL10, TNFa, and VEGF) were not changed in SOCS3-deficient myeloid cells. The role of Gas6 in the regulation of angiogenic processes has been discussed in several studies. On the one side, Gas6 promotes angiogenesis by stimulation of endothelial cell proliferation and migration [41]. On the other side, Gallicchio et al. [42] demonstrated that the stimulation of the tyrosine kinase receptor Axl by Gas6 in endothelial cells results in the inhibition of the VEGFdependent activation of the VEGFR-2 resulting in the inhibition of VEGF-mediated angiogenesis. The currently 
existing discrepancies might be explained by the involvement of Gas6 in multiple cellular processes (e.g., phagocytosis, cell adhesion, migration, proliferation, and inflammatory cytokine release) mediated through different corresponding Tyro-3, Axl, and Mer (TAM) receptor tyrosine kinases [43]. TAM receptors are essential regulators of immune homeostasis by the recognition and phagocytosis of PS-exposing apoptotic cells via their ligands Gas6 and Protein S [44]. In addition, all 3 TAM receptors are able to promote other cellular functions, such as cell survival, through mechanisms involving, for example, the PI3K/Akt or ERK1/2 signaling pathway or proliferation and migration [45], and they were shown to be expressed in endothelial cells [41, 42, 46]. Taken together, the impact of Gas6 on the individual TAM receptors either acting cooperatively or by distinct signaling pathways on diverse functions of angiogenesis needs to be further addressed. And although our data strongly indicate that Gas6 improves phagocytic functions of microglia/macrophages and by this diminishes pathological neovascularization, as observed in the ex vivo aortic ring assay, we appreciate that an elevated Gas6 production by myeloid cells might additionally stimulate the prosurvival signaling, proliferation, and migration in some endothelial cells. Based on our findings, future investigations in in vivo models are necessary, addressing whether the specific modulation of phagocytic function of myeloid cells via Gas6 might represent a promising therapeutic tool for interventions on pathological angiogenesis.

\section{Acknowledgments}

We thank Prof. Dr. Triantafyllos Chavakis for thoughtful discussions and critical reading the manuscript. We are grateful for the technical assistance of Bettina Gercken, Silvia Großklaus, Marta Prucnal, Janine Gebler, Christine Mund, Carmen Friebel, and Bianca Müller.

\section{Statement of Ethics}

Animal experiments conform to internationally accepted standards and have been approved by the Landesdirektion Sachsen, Germany.

\section{Disclosure Statement}

The authors have no conflicts of interest to declare.

\section{Author Contributions}

I.K. and A.K.A.: designed and performed the experiments, analyzed and interpreted the data, and wrote the manuscript. A.N., M.T., and D.S.: performed experiments and analyzed data. D.M.P.: provided reagents, technical advice, and supported the work with helpful discussions. A.D.: helped to write the manuscript.

\section{References}

1 Morioka S, Maueröder C, Ravichandran KS. Living on the Edge: Efferocytosis at the Interface of Homeostasis and Pathology. Immunity. 2019 May;50(5):1149-62.

2 Gheibi Hayat SM, Bianconi V, Pirro M, Sahebkar A. Efferocytosis: molecular mechanisms and pathophysiological perspectives. Immunol Cell Biol. 2019 Feb;97(2):124-33.

3 Elliott MR, Koster KM, Murphy PS. Efferocytosis Signaling in the Regulation of Macrophage Inflammatory Responses. J Immunol. 2017 Feb;198(4):1387-94.

4 Klotzsche-von Ameln A, Cremer S, Hoffmann J, Schuster P, Khedr S, Korovina I, et al. Endogenous developmental endothelial locus-1 limits ischaemia-related angiogenesis by blocking inflammation. Thromb Haemost. 2017 Jun;117(6):1150-63.

5 Murdoch C, Muthana M, Coffelt SB, Lewis CE. The role of myeloid cells in the promotion of tumour angiogenesis. Nat Rev Cancer. 2008 Aug;8(8):618-31.
6 Gurevich DB, Severn CE, Twomey C, Greenhough A, Cash J, Toye AM, et al. Live imaging of wound angiogenesis reveals macrophage orchestrated vessel sprouting and regression. EMBO J. 2018 Jul;37(13):e97786.

7 Fantin A, Vieira JM, Gestri G, Denti L, Schwarz Q, Prykhozhij S, et al. Tissue macrophages act as cellular chaperones for vascular anastomosis downstream of VEGF-mediated endothelial tip cell induction. Blood. 2010 Aug;116(5):829-40.

8 Baer C, Squadrito ML, Iruela-Arispe ML, De Palma M. Reciprocal interactions between endothelial cells and macrophages in angiogenic vascular niches. Exp Cell Res. 2013 Jul; 319(11):1626-34.

9 Altmann C, Schmidt MH. The Role of Microglia in Diabetic Retinopathy: Inflammation, Microvasculature Defects and Neurodegeneration. Int J Mol Sci. 2018 Jan;19(1):E110.
10 Abcouwer SF. Neural inflammation and the microglial response in diabetic retinopathy. $J$ Ocul Biol Dis Infor. 2012 Apr;4(1-2):25-33.

11 Korovina I, Neuwirth A, Sprott D, Weber S, Sardar Pasha SP, Gercken B, et al. Hematopoietic hypoxia-inducible factor $2 a$ deficiency ameliorates pathological retinal neovascularization via modulation of endothelial cell apoptosis. FASEB J. 2019 Feb;33(2):1758-70.

12 Poché RA, Hsu CW, McElwee ML, Burns AR, Dickinson ME. Macrophages engulf endothelial cell membrane particles preceding pupillary membrane capillary regression. Dev Biol. 2015 Jul;403(1):30-42.

13 Yoshimura A, Naka T, Kubo M. SOCS proteins, cytokine signalling and immune regulation. Nat Rev Immunol. 2007 Jun;7(6):454-65.

14 Qin H, Holdbrooks AT, Liu Y, Reynolds SL, Yanagisawa LL, Benveniste EN. SOCS3 deficiency promotes M1 macrophage polarization and inflammation. J Immunol. $2012 \mathrm{Oct}$; 189(7):3439-48. 
15 Arnold CE, Whyte CS, Gordon P, Barker RN, Rees AJ, Wilson HM. A critical role for suppressor of cytokine signalling 3 in promoting M1 macrophage activation and function in vitro and in vivo. Immunology. 2014 Jan; 141(1):96-110.

16 Mahony R, Ahmed S, Diskin C, Stevenson NJ. SOCS3 revisited: a broad regulator of disease, now ready for therapeutic use? Cell Mol Life Sci. 2016 Sep;73(17):3323-36.

17 Gordon P, Okai B, Hoare JI, Erwig LP, Wilson HM. SOCS3 is a modulator of human macrophage phagocytosis. J Leukoc Biol. 2016 Oct; 100(4):771-80.

18 Clausen BE, Burkhardt C, Reith W, Renkawitz R, Förster I. Conditional gene targeting in macrophages and granulocytes using LysMcre mice. Transgenic Res. 1999 Aug; 8(4):265-77.

19 Yasukawa H, Ohishi M, Mori H, Murakami M, Chinen T, Aki D, et al. IL-6 induces an anti-inflammatory response in the absence of SOCS3 in macrophages. Nat Immunol. 2003 Jun;4(6):551-6.

20 Baker M, Robinson SD, Lechertier T, Barber PR, Tavora B, D'Amico G, et al. Use of the mouse aortic ring assay to study angiogenesis. Nat Protoc. 2011 Dec;7(1):89-104.

21 Schindelin J, Arganda-Carreras I, Frise E, Kaynig V, Longair M, Pietzsch T, et al. Fiji: an open-source platform for biological-image analysis. Nat Methods. 2012 Jun;9(7):676-82.

22 Zudaire E, Gambardella L, Kurcz C, Vermeren S. A computational tool for quantitative analysis of vascular networks. PLoS One. 2011;6(11):e27385.

23 Johnson J. Not seeing is not believing: improving the visibility of your fluorescence images. Mol Biol Cell. 2012 Mar;23(5):754-7.

24 Moussaud S, Draheim HJ. A new method to isolate microglia from adult mice and culture them for an extended period of time. J Neurosci Methods. 2010 Mar;187(2):243-53.

25 Chung KJ, Chatzigeorgiou A, Economopoulou M, Garcia-Martin R, Alexaki VI, Mitroulis I, et al. A self-sustained loop of inflammation-driven inhibition of beige adipogenesis in obesity. Nat Immunol. 2017 Jun;18(6): 654-64.
26 Belmokhtar CA, Hillion J, Ségal-Bendirdjian E. Staurosporine induces apoptosis through both caspase-dependent and caspase-independent mechanisms. Oncogene. 2001 Jun; 20(26):3354-62.

27 Livak KJ, Schmittgen TD. Analysis of relative gene expression data using real-time quantitative PCR and the 2(-Delta Delta C(T)) Method. Methods. 2001 Dec;25(4):402-8.

28 Ishimoto Y, Ohashi K, Mizuno K, Nakano T. Promotion of the uptake of PS liposomes and apoptotic cells by a product of growth arrestspecific gene, gas6. J Biochem. 2000 Mar; 127(3):411-7.

29 Shao WH, Zhen Y, Eisenberg RA, Cohen PL. The Mer receptor tyrosine kinase is expressed on discrete macrophage subpopulations and mainly uses Gas6 as its ligand for uptake of apoptotic cells. Clin Immunol. 2009 Oct; 133(1):138-44.

30 Sather S, Kenyon KD, Lefkowitz JB, Liang X, Varnum BC, Henson PM, et al. A soluble form of the Mer receptor tyrosine kinase inhibits macrophage clearance of apoptotic cells and platelet aggregation. Blood. 2007 Feb; 109(3):1026-33.

31 Ballantine L, Midgley A, Harris D, Richards E, Burgess S, Beresford MW. Increased soluble phagocytic receptors sMer, sTyro3 and sAxl and reduced phagocytosis in juvenile-onset systemic lupus erythematosus. Pediatr Rheumatol Online J. 2015 Apr;13(1):10.

32 Gelati M, Aplin AC, Fogel E, Smith KD, Nicosia RF. The angiogenic response of the aorta to injury and inflammatory cytokines requires macrophages. J Immunol. 2008 Oct; 181(8):5711-9.

33 Nicosia RF. The aortic ring model of angiogenesis: a quarter century of search and discovery. J Cell Mol Med. 2009 Oct;13(10): 4113-36.

34 Ligresti G, Aplin AC, Zorzi P, Morishita A, Nicosia RF. Macrophage-derived tumor necrosis factor-alpha is an early component of the molecular cascade leading to angiogenesis in response to aortic injury. Arterioscler Thromb Vasc Biol. 2011 May;31(5):1151-9.

35 Winn RK, Harlan JM. The role of endothelial cell apoptosis in inflammatory and immune diseases. J Thromb Haemost. 2005 Aug;3(8): 1815-24.
36 Zhao X, Liao Y, Morgan S, Mathur R, Feustel $\mathrm{P}$, Mazurkiewicz J, et al. Noninflammatory Changes of Microglia Are Sufficient to Cause Epilepsy. Cell Rep. 2018 Feb;22(8):2080-93.

37 Carow B, Rottenberg ME. SOCS3, a Major Regulator of Infection and Inflammation. Front Immunol. 2014 Feb;5:58.

38 Rouillard AD, Gundersen GW, Fernandez NF, Wang Z, Monteiro CD, McDermott MG, et al. The harmonizome: a collection of processed datasets gathered to serve and mine knowledge about genes and proteins. Database (Oxford). 2016;2016:pii:baw100.

39 Santulli-Marotto S, Gervais A, Fisher J, Strake B, Ogden CA, Riveley C, et al. Discovering Molecules That Regulate Efferocytosis Using Primary Human Macrophages and High Content Imaging. PLoS One. 2015 Dec; 10(12):e0145078.

40 Watson EC, Grant ZL, Coultas L. Endothelial cell apoptosis in angiogenesis and vessel regression. Cell Mol Life Sci. 2017 Dec;74(24): 4387-403.

41 Kim YS, Jung SH, Jung DH, Choi SJ, Lee YR, Kim JS. Gas6 stimulates angiogenesis of human retinal endothelial cells and of zebrafish embryos via ERK1/2 signaling. PLoS One. 2014 Jan;9(1):e83901.

42 Gallicchio M, Mitola S, Valdembri D, Fantozzi R, Varnum B, Avanzi GC, et al. Inhibition of vascular endothelial growth factor receptor 2-mediated endothelial cell activation by Axl tyrosine kinase receptor. Blood. 2005 Mar;105(5):1970-6.

43 van der Meer JH, van der Poll T, van 't Veer C. TAM receptors, Gas6, and protein S: roles in inflammation and hemostasis. Blood. 2014 Apr;123(16):2460-9.

44 Nagata S. Apoptosis and Clearance of Apoptotic Cells. Annu Rev Immunol. 2018 Apr; 36(1):489-517.

45 Lemke G. Biology of the TAM receptors. Cold Spring Harb Perspect Biol. 2013 Nov; 5(11):a009076

46 Zhen Y, Finkelman FD, Shao WH. Mechanism of Mer receptor tyrosine kinase inhibition of glomerular endothelial cell inflammation. J Leukoc Biol. 2018 Apr;103(4):709-17. 